\title{
A Novel Network Delay Based Side Channel Attack: Modeling and Defense
}

\author{
Zhen Ling*, Junzhou Luo*, Yang Zhang*, Xinwen $\mathrm{Fu}^{\dagger}$ and Wei $\mathrm{Yu}^{\ddagger}$ \\ *Southeast University, Nanjing 211189, P. R. China. zhenling@seu.edu.cn \\ ${ }^{\dagger}$ University of Massachusetts Lowell, Lowell, MA 01854, USA. xinwenfu@cs.uml.edu \\ ‡Towson University, Towson, MD 21252, USA. wyu@ towson.edu
}

\begin{abstract}
Information leakage via side channels has become a primary security threat to encrypted web traffic. Existing side channel attacks and corresponding countermeasures focus primarily on packet length, packet timing, web object size and web flow size. However, we found that encrypted web traffic can also leak information via network delay between a user and the web sites she visits. Motivated by this observation, we investigate a novel network-delay based side-channel attack to infer web sites visited by a user. The adversary can utilize pattern recognition techniques to differentiate web sites by measuring sample mean and sample variance of the round-trip time (RTT) between a victim user and web sites. We theoretically analyzed the damage caused by such an adversary and derived closed-form formulas for detection rate, the probability that the adversary correctly recognizes a web site. To defeat this side channel attack, we proposed several countermeasures. The basic idea is to shape traffic from different web sites so that they have similar RTT statistics. We proposed strategies based on the $k$ means clustering and $\mathbb{K}$-Anonymity to ensure that traffic shaping will not cause excessive delay while providing a predictable degree of anonymity. We conducted extensive experiments and our empirical results match our theory very well.
\end{abstract}

Index Terms-Network Delay, Side Channel, Information Leak, Countermeasures

\section{INTRODUCTION}

With growing concerns over privacy, security and censorship, various privacy enhancing technologies (PET) have been proposed and deployed to improve users' Internet experience [1]. Encrypted tunnels is one common strategy to hide the content and IP addresses of web sites from third-party observers. Single-hop systems including OpenSSH tunnels, simple SSL proxies and virtual private networks (VPN) are examples of encrypted tunnels. NAT enabled wireless routers with WPA/WPA2 encryption also provide anonymity and privacy for users behind these routers to some extent. However, these technologies are insufficient to prevent a third party from taking advantage of side channels to infer critical information from encrypted web traffic and identifying web sites being visited by users.

To identify web sites visited by users, an adversary can profile the "fingerprints" of these web sites. Side channels provide such a fingerprint, and are often based on statistics of web traffic. Existing side channel attacks against webs can be classified into two categories: object based and packet based techniques. In web object based side channels [2], an adversary explores the HTTP response and estimates the size of HTML objects, and then measures the similarity between the estimated feature and profile database. In packet based side channels [3], [4], [5], [6], [7], the adversary takes advantage of features of encrypted packets, including the packet size distribution, flow direction, packet inter-arrival times and packet ordering. To prevent information leakage from these side channels, researchers have proposed two categories of countermeasures: server-side solution [8] and client-side solution [9]. A server-side solution morphs traffic from different web sites so that they look similar to each other. The clientside countermeasure against side channels manipulates TCP and HTTP features of web traffic.

In this paper, we investigate a novel network delay based side channel attack against encrypted web traffic. Through the study of web traffic via the SSH tunnel, we found that network delay, i.e., the round-trip time (RTT), could leak critical information about the encrypted web traffic. Motivated by this observation, we adopt information theory to estimate the information leakage from RTTs, and establish a generic traffic classification model to identify web sites accessed by users. Sample mean and sample variance are used as statistical features of RTTs to differentiate web sites. Detection rate is defined as the probability that an adversary can correctly recognize a web site. We derive closed-form formulas for the detection rate in terms of parameters including the mean and variance of the network delay and the sample size.

To counter the attack, we proposed three defense strategies: (i) In the naive statistical distribution based approach, we manipulate the web traffic delay at the proxy side so that sample mean and sample variance of the RTT for all web sites are similar. However, this strategy introduces significant delay into web traffic. (ii) In the $k$-means clustering based strategy, we classify web sites into several clusters and manipulate web traffic from each cluster of web sites so that their sample mean and sample variance are similar. However, the $k$-means clustering cannot predict the number of web sites in each cluster and cannot guarantee the degree of anonymity for these clusters of web sites. (iii) To guarantee the degree of anonymity for each cluster of web sites, we propose the $\mathbb{K}$ Anonymity based approach. An efficient heuristic algorithm is proposed to adjust the number of web sites in each cluster to achieve $\mathbb{K}$-Anonymity, i.e., at least $\mathbb{K}$ web sites in each cluster. This $\mathbb{K}$-Anonymity strategy provides the tradeoff between privacy and web surfing experience.

We conducted extensive real-world experiments to validate the severity of the novel side channel attack and the feasibility 
and effectiveness of our countermeasures. Our experiments demonstrate that the $\mathbb{K}$-Anonymity based strategy can effectively balance the tradeoff between the performance and information leakage. Our data match our theory very well.

The remainder of this paper is organized as follows: We review the related work in Section II, and present the network delay based side channel attack, including network and threat models, and information leakage metric in Section III. In Section IV, we analyze the detection rate and derive the closed formulas for detection rate when the adversary uses sample mean and sample variance as the statistical features. We propose the countermeasures in Section V. Extensive experimental results are presented in Section VI and we conclude this paper in Section VII.

\section{RELATED WORK}

Existing traffic analysis techniques can largely be categorized into two groups: side channels (passively observing traffic) and covert channels (actively manipulating traffic). In a side channel attack, an attacker records traffic passively and identifies the similarity between server's outbound traffic and client's inbound traffic to correlate the communication relationship. For example, Zhu et al. [10] used mutual information for the similarity measurement. Levine et al. [11] used a cross correlation technique. Research also showed that the attacker could infer sensitive information from encrypted network traffic by examining patterns of packet size and timing. For example, Song et al. [12] utilized the packet inter-arrival timing in SSHv1 connections to infer keystroke patterns and ultimately crack the typed passwords. Sun et al. [13] investigated the sizes of HTML objects transmitted over SSL connections and were able to identify the web pages based on the number and size of objects in each encrypted HTTP response. Liberatore and Levine [4] examined packet size of HTTP traffic transmitted over the persistent connection or tunneled via SSH that could statistically identify the web pages. Lu et al. [6] improved the web site fingerprinting techniques by utilizing packet size ordering information. Wright et al. [14] investigated the packet size statistical distribution in encrypted Voice over IP (VoIP) connections and could identify the spoken language. Later work of Wright et al. [15] also investigated how an eavesdropper could identify spoken phrases in encrypted VoIP.

The covert channel techniques intend to embed a specific secret signal into target traffic. Such techniques can reduce the false positive rate significantly if the signal is long enough and does not require massive traffic training as required in side channel techniques. For example, Wang et al. [16] proposed an active watermarking scheme that was robust to random timing perturbation. They analyzed the tradeoffs between the true positive rate, the maximum timing perturbation added by attackers, and the number of packets needed to successfully decode the watermark. Wang et al. [17] investigated the feasibility of a timing-based watermarking scheme to identify the encrypted peer-to-peer VoIP calls. By slightly changing the timing of packets, their approach can correlate encrypted anonymous network connections. Peng et al. [18] analyzed the secrecy of timing-based watermarking techniques proposed in [16], based on the distribution of traffic timing. Yu et al. [19] proposed a flow marking scheme based on the direct sequence spread spectrum (DSSS) technique. This technique could be used by attackers to secretly confirm the communication relationship via mix networks. Ling et al. [20] proposed the cell counter based attack against Tor, in which an attacker embeds a signal into the variation of cell counter of the target traffic at the malicious exit onion router. Ling et al. [21] enhanced the approach without controlling the entry and exit node. Ling et al. [22] investigated the least significant packet size based attack against Anonymizer.

To counter traffic analysis attacks, Wright et al. [8] proposed a server-side based traffic morphing approach in which they modulate the source packet size so that packet size distributions in different traffic flows are similar to each other [14], [4]. However, this technique is vulnerable to the enhanced web fingerprinting techniques [6]. Luo et al. [9] investigated a client-side based composite traffic transformation techniques by exploiting the basic protocol features of TCP and HTTP to manipulate the characteristics of encrypted web traffic. Unfortunately, the authors did not consider the side channel technique caused by the RTTs between clients and web sites. This paper addresses the RTT based side channel.

\section{A Network Delay Based Side Channel Attack}

In this section, we first present network and threat models. We then introduce the novel side-channel attack via network delay against encrypted web traffic for interring the corresponding web sites.

\section{A. Network and Threat Models}

Figure 1 gives our network model and threat model. It has four participants: a victim client, an attacker, a remote proxy and web sites. The client connects to a remote proxy over an encrypted transport layer. The proxy makes requests on behalf of the client, and return the results over the encrypted connection to the client. We assume that the adversary is capable of recording the timestamp and length of packets. In addition, we assume that the proxy does not intentionally introduce additional delay and perturb the passing traffic. The proxy that we use to exemplify the side channel attack is a SOCKS based OpenSSH implementation. However, network delay based side channel attacks can be applied against VPN proxies and WEP/WPA wireless routers as well.

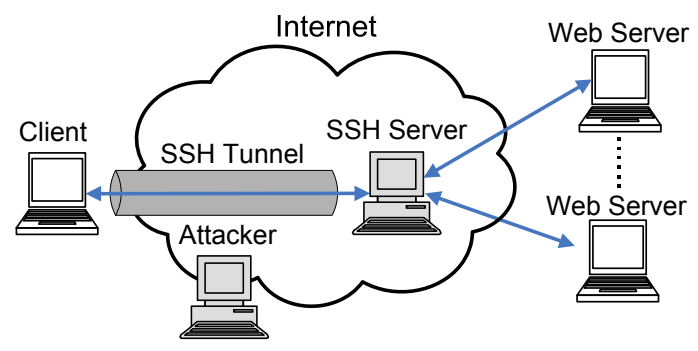

Fig. 1. Network Model and Threat Model 
In order to measure RTT between a victim user and a remote web site, the adversary observes the communication and utilize appropriate packets to derive RTT. After a client is authenticated by the proxy, a SSH tunnel is built between the client and proxy. When the web browser accesses the web site through the local SOCKS proxy, the client sends a "SSH_MSG_CHANNEL_OPEN" packet to the SSH proxy. The proxy receives the packet and initiates a TCP connection to the web site on behalf of the client. Once the connection is established, the proxy sends back a "SSH_MSG_CHANNEL_OPEN_CONFIRMATION" packet to the client. Subsequently, the client transmits an HTTP "GET" packet to retrieve the web object and the proxy forwards the packet to web site. Then web site sends back the HTTP response packet.

This basic workflow is illustrated in Figure 2. We assume the client $C$ accesses the remote web site $W$ via the proxy $P$. Denote the RTT between the client $C$ and proxy $P$ as $T_{C P}$, and denote the RTT between the proxy $P$ and web site $W$ as $T_{P W}$. Assume the packet processing time at proxy is $T_{P}$. Then the RTT between client $C$ and web site $W$ can be written as follows,

$$
T_{C W}=T_{C P}+T_{P}+T_{P W} .
$$

Our threat model is also illustrated in Figure 2. To derive $T_{C W}$, the adversary can measure the time lapse between the HTTP "GET" and the corresponding response. In this way, the adversary is capable of building RTT profiles between a client and a list of known web sites. By using these profiles, the adversary attempts to decide, to some extent, which of the known RTT most closely resembles the encrypted, unknown RTT. The technique will be further explained in details in Section III-C.

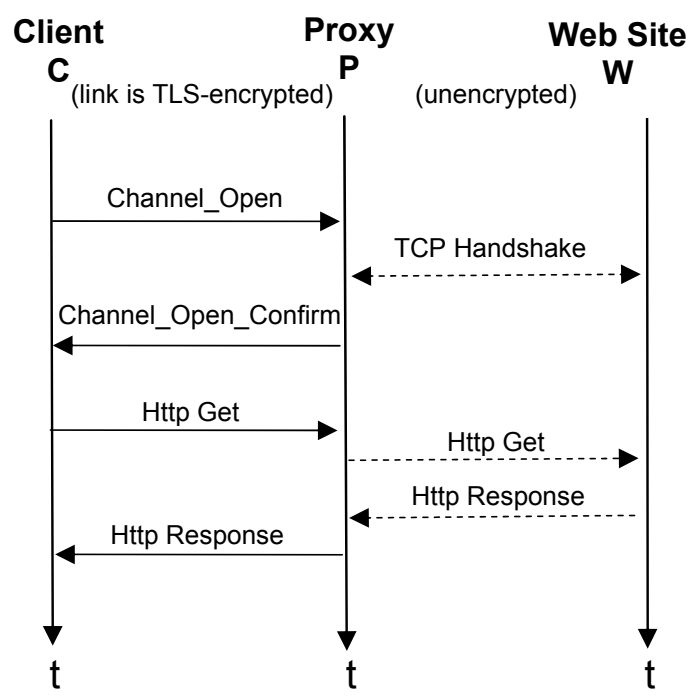

Fig. 2. Workflow of SSH Connection Establishment

\section{B. Information Leakage Metrics}

We adopt the information theoretic metrics to estimate the information leakage via the RTT based side channel. To define anonymity, we adopt the definition by Pfitzmann and Köhntopp in [23]. Anonymity is the state of being not identifiable within a set of subjects, the anonymity set. A sender is identifiable when we get information that can be linked to him. In our context, the "sender" is the web site a client is accessing. Hence, we only consider the sender's anonymity in this paper. We consider an adversary who has obtained the profiles of different websites a client may visit. Anonymity degree is the probability that the adversary recognizes the correct web site that the client is visiting. Denote the potential web sites that the client accesses as $\left\{W_{1}, W_{2}, \ldots, W_{N}\right\}$, where $N$ is the total number of web site profiles. Given sample RTT $r$ measured by the adversary, the adversary attempts to identify the web sites based on the offline learned RTT profiles of these web sites. The probability that the client accesses a web site $W_{i}$ is $P\left(W_{i} \mid r\right)$. Therefore, the uncertain information after the adversary makes a RTT measurement, anonymity degree, is defined as

$$
H=-\sum_{i=1}^{N} P\left(W_{i} \mid r\right) \log _{2}\left(P\left(W_{i} \mid r\right)\right) .
$$

The information leakage is related to the probabilities that a client visits individual web sites. Without loss of generality, we assume that a client visits each web site with equal probability. This is a worst case for the adversary since the uncertainty that a client visits different web sites is maximum in this case, i.e., $H_{M}=\log N$. Therefore, the information leakage $H_{A}$ to the adversary is

$$
H_{A}=H_{M}-H
$$

We give a simple example to explain the information leakage. Suppose a client $C$ is visiting web site $W_{i}$. The RTT between the client and web site is $T_{C W_{i}}$. Denote the mean and standard deviation of RTT $T_{C W_{i}}$ as $M\left(T_{C W_{i}}\right)$ and $D\left(T_{C W_{i}}\right)$, respectively. The scope of the RTT $T_{C W_{i}}$ is defined as $S\left(T_{C W_{i}}\right):\left[M\left(T_{C W_{i}}\right)-D\left(T_{C W_{i}}\right), M\left(T_{C W_{i}}\right)+D\left(T_{C W_{i}}\right)\right]$. Denote the number of other web sites $W_{j}(1 \leqslant j \leqslant N, j \neq$ i) whose scopes overlap with $S\left(T_{C W_{i}}\right)$ as $N_{W}$. Then the probability that the client is accessing the web sites $W_{i}$ is $P\left(W_{j} \mid T_{C W_{i}}\right)=\frac{1}{N_{W}}$. Consequently, according to Equations (2) and (3), the leakage information can be indicated as $H_{A}=H_{M}-H=\log N-\log N_{W}$.

\section{Attacking Strategy}

Recall that the goal of the adversary is to infer which of the $N$ possible web sites is visited by the client, and the adversary can measure the RTTs between the client and remote web sites by observing the traffic. Assume that the adversary adopts the pattern recognition strategy based on Bayes decision theory. The attack has two phases: Off-line profiling and run-time classification as follows.

Phase 1: Off-line profiling The off-line profiling consists of the following procedures:

1) The adversary selects a statistical feature of the RTT for web site classification. Possible features we study in this paper are sample mean and sample variance. 
2) The kernel estimator of a density function with kernel $G$ is defined by

$$
f(s)=\frac{1}{M h} \sum_{i=1}^{M} G\left(\frac{s-S_{i}}{h}\right),
$$

where $h$ is the window width, also called the smoothing parameter or bandwidth, $S_{i}$ is the $i^{\text {th }}$ observation of the network delay, $M$ is the number of such observations, and $G($.$) is based on Gaussian kernel, i.e., G(s)=$ $\frac{1}{\sqrt{2 \pi}} \exp \left(-\frac{s^{2}}{2}\right)$.

3) Based on the PDFs of statistical features for different web sites, Bayes decision rules are derived. Recall that there are $N$ possible web sites $W_{1}, \ldots, W_{m}$. The Bayes decision rule can be stated as follows:

The sample is classified as one from web site $w_{i}$ if

$$
P\left(W_{i} \mid s\right) \geqslant P\left(W_{j} \mid s\right) .
$$

That is,

$$
f\left(s \mid W_{i}\right) P\left(W_{i}\right) \geqslant f\left(s \mid W_{j}\right) P\left(W_{j}\right),
$$

for all $j=1, \ldots, N$. Here $P\left(W_{i}\right)$ is a priori probability that the web site $W_{i}$ can be accessed, and $P\left(W_{i} \mid s\right)$ is the post priori probability that the web site $W_{i}$ is accessed by the user when the collected sample has the measured feature $s$.

Phase 2: Run-time Classification Once the profiling phase is complete, the adversary is ready to start the classification at run time. The method in Section III-A can be used to collect a sample of RTT between the client and remote web sites. Then the adversary calculates the value of the statistical feature from the collected sample, and utilizes the Bayes decision rules derived in the profiling phase to match the collected sample to one of the known web sites.

\section{ATTACK ANALYSIS}

In this section, we analyze the effectiveness of the network delay based side channel attack in terms of detection rate. We first formally define the detection rate as the probability that the adversary can correctly classify the target traffic, that is, identify the web site. We then derive the closed-form formulas of detection rate when the adversary uses sample mean and sample variance as the statistical feature respectively. Our formulas will be approximate ones due to the complexity of the problem. However, these formulas do correctly reflect the impact of the side channel attack, including the sample size and statistical feature used. The theoretical analysis plays a critical important role in the design of countermeasures in Section V.

\section{A. Bayes Decision Rule}

Without loss of generality, we analyze the classification of two web sites, $W_{i}$ and $W_{j}$. Figure 3 shows the PDFs of the statistical features conditioned on the two web sites. Let $d$ be the solution of the following equation,

$$
f\left(W_{i} \mid s\right)=f\left(W_{j} \mid s\right)
$$

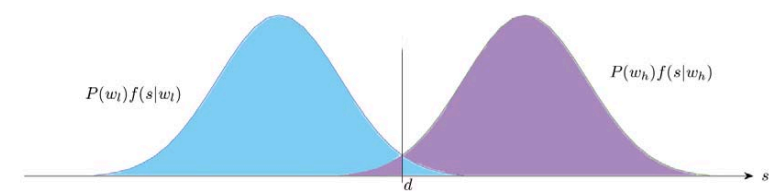

Fig. 3. Bayes Decision Making for the Case of Two Web Sites

Assume that there is a unique solution to the equation. The Bayes decision rule now becomes

$$
\begin{gathered}
\text { If } s \leqslant d, \text { the web site is } W_{i} \\
\text { otherwise, the web site is } W_{j} .
\end{gathered}
$$

The error rate $P_{e}$ can then be calculated by

$$
P_{e}=P\left(W_{i}\right) \int_{-\infty}^{d} f\left(s \mid W_{i}\right) d s+P\left(W_{j}\right) \int_{d}^{+\infty} f\left(s \mid W_{j}\right) d s .
$$

The detection rate is given by

$$
\begin{aligned}
P_{D}=1-P_{e} & =P\left(W_{i}\right) \int_{d}^{+\infty} f\left(s \mid W_{i}\right) d s \\
& +P\left(W_{j}\right) \int_{-\infty}^{d} f\left(s \mid W_{j}\right) d s .
\end{aligned}
$$

We use three distinct features, that is, sample mean, sample variance, and the vector of sample mean and sample variance for the classification. By using these features, the adversary carries out the attack and our experimental results shown in Section VI demonstrate that the RTT based side channel attack is a severe threat to user privacy while existing defense techniques cannot address this challenge.

\section{B. Decomposition of the RTT}

We formally analyze the composition of the RTT between a user and the web sites. Recall that the adversary collects a sample of RTT at run time in order to perform the classification. To derive detection rate, we need to formally model RTT. Let random variable $T$ be the RTT between the client and remote web sites. Let $T_{c}$ be the RTT between the client and the proxy and $T_{w_{x}}$ be the RTT between the proxy and remote web site $w_{x}$. Let $T_{p}$ be the queueing time of the proxy. Then the RTT $T$ can be derived by

$$
T=T_{c}+T_{p}+T_{w_{x}}
$$

We assume that $T_{c}, T_{p}$ and $T_{w_{x}}$ are normally distributed. Notice that this assumption simplifies our analysis without loss of generality and will be validated by our experiments in Section VI. Therefore,

$$
\begin{aligned}
T_{c} & \sim \mathcal{N}\left(\mu_{c}, \sigma_{c}^{2}\right), \\
T_{p} & \sim \mathcal{N}\left(\mu_{p}, \sigma_{p}^{2}\right), \\
T_{w_{x}} & \sim \mathcal{N}\left(\mu_{w_{x}}, \sigma_{w_{x}}^{2}\right) .
\end{aligned}
$$

Denote $T_{i}$ and $T_{j}$ as RTT random variables when a client visits web sites $w_{i}$ and $w_{j}$ respectively. Denote $\mu_{i}$ and $\mu_{j}$ as the mean of $T_{i}$ and $T_{j} . \sigma_{i}$ and $\sigma_{j}$ are the variance of $T_{i}$ and $T_{j}$. We have

$$
T_{i} \sim \mathcal{N}\left(\mu_{i}, \sigma_{i}^{2}\right),
$$


where $\mu_{i}=\mu_{c}+\mu_{p}+\mu_{w_{i}}$ and

$$
\begin{gathered}
\sigma_{i}^{2}=\sigma_{c}^{2}+\sigma_{p}^{2}+\sigma_{w_{i}}^{2} . \\
T_{j} \sim \mathcal{N}\left(\mu_{j}, \sigma_{j}^{2}\right),
\end{gathered}
$$

where $\mu_{j}=\mu_{c}+\mu_{p}+\mu_{w_{j}}$ and

$$
\sigma_{j}^{2}=\sigma_{c}^{2}+\sigma_{p}^{2}+\sigma_{w_{j}}^{2} .
$$

We also introduce the ratio

$$
r=\frac{\sigma_{i}^{2}}{\sigma_{j}^{2}}=\frac{\sigma_{c}^{2}+\sigma_{p}^{2}+\sigma_{w_{i}}^{2}}{\sigma_{c}^{2}+\sigma_{p}^{2}+\sigma_{w_{j}}^{2}}
$$

where $\sigma_{c}, \sigma_{p}, \sigma_{w_{i}}$ and $\sigma_{w_{j}}$ are defined in Equations (12), (13), (16), (18), respectively.

Based on the results in Section VI, we conclude that sample mean and sample variance of the RTTs can be used to identify the web sites accessed by users effectively.

\section{Detection Rate}

1) The Case of Sample Mean: Denote $\left\{X_{1}, X_{2}, \ldots, X_{n}\right\}$ as a sample of RTT. The sample mean is the average of the elements in the sample, then we can get

$$
\bar{X}=\frac{\sum_{i=1}^{n} X_{i}}{n} .
$$

Notice that sample mean $\bar{X}$ is a random variable, and an unbiased estimation of $X$ 's mean $\mu$. Then we derive a closedform formula for the estimation of detection rate when the adversary uses sample mean as the feature statistic, which is stated in Theorem 1. The detailed proof is in Appendix A.

Theorem 1. When sample mean is used as the feature statistic, the detection rate can be estimated by

$$
P_{d}(\bar{x}) \approx 1-e^{-\left(\mu_{j}-\mu_{i}\right)^{2} / 4} e^{-\frac{n}{\sigma_{i}^{2}+\sigma_{j}^{2}}} \frac{1}{\sqrt{2\left(\frac{1}{\sqrt{r}}+\sqrt{r}\right)}},
$$

where $r$ is defined in Equation (19).

From Theorem 1 the following observations can be made:

- Detection rate in Equation (21) is increasing with sample size $n$. That is, if an adversary can collect a large enough sample of RTT from the same web site, the web site will be disclosed at last if different web sites have different means of RTT.

- Detection rate $P_{d}(\bar{x})$ is an increasing function of the difference of means $\mu_{j}-\mu_{i}$ of two web sites. If two web sites have different RTT means, the adversary can use sample mean to easily differentiate them. Later our experiments will demonstrate the significance of this observation.
2) The Case of Sample Variance: Denote $\left\{X_{1}, X_{2}, \ldots, X_{n}\right\}$ as a random sample of RTT of size $n$ from the distribution $X$. The sample variance $Y$ is defined by

$$
Y=\frac{\sum_{i=1}^{n}\left(X_{i}-\bar{X}\right)^{2}}{n-1} .
$$

Notice sample variance $Y$ is a random variable, and an unbiased estimation of $X$ 's variance. Then we derive a closed-form formula based on Chebyshev inequality for the estimation of detection rate when the adversary uses sample variance of RTT as the feature statistic. The estimation of detection rate can be derived by the following theorem. The detailed proof of Theorem 2 can be found in Appendix B of our technical report [24].

Theorem 2. Using sample variance of sample size $n$ as the classification feature gives rise to an estimated detection rate $P_{d}(Y)$ as

$$
P_{d}(Y) \approx \max \left(1-\frac{V_{Y}}{n-1}, 50 \%\right),
$$

where $V_{Y}$ is calculated in Equation (24).

$$
V_{Y}=\frac{1}{2\left(1-\frac{\ln r}{r-1}\right)^{2}}+\frac{1}{2\left(\frac{r \ln r}{r-1}-1\right)^{2}},
$$

and $r=\frac{\sigma_{i}^{2}}{\sigma_{j}^{2}}$ as define in Equation (19).

From Theorem 2, the following observations can be made:

- Detection rate $P_{d}(Y)$ is an increasing function of sample size $n$. When $n \rightarrow \infty$, detection rate $P_{d}(Y) \rightarrow 100 \%$. This implies that the more samples an adversary could collect, the higher detection rate via sample variance of RTT it can achieve.

- As shown in the second part of Proof of Theorem 2 in Appendix B of of our technical report [24], detection rate $P_{d}(Y)$ is an increasing function of $r$. That is, the smaller $r$, the lower detection rate. When $r=1$, detection rate is $50 \%$. This corresponds to the case when random delay with sufficiently large $\sigma_{p}^{2}$ is applied to the web traffic. This suggests that although the adversary may use a big size of sample to infer web sites by sample variance, extra random delay can make such an attack infeasible.

\section{Countermeasure}

In this section, we introduce countermeasures to mitigate the threat from the RTT based side channel. We start with a naive approach, followed by $k$-means clustering based approach and $\mathbb{K}$-Anonymity based approach.

\section{A. Naive Countermeasure}

According to Shannon's perfect secrecy principle, if the distribution of all the RTTs between the client and each web sites are similar to one another, the information gained from the attack approaches zero and the anonymity degree reaches maximum. Following this principle, we develop the statistical distribution based countermeasure. We select a web 


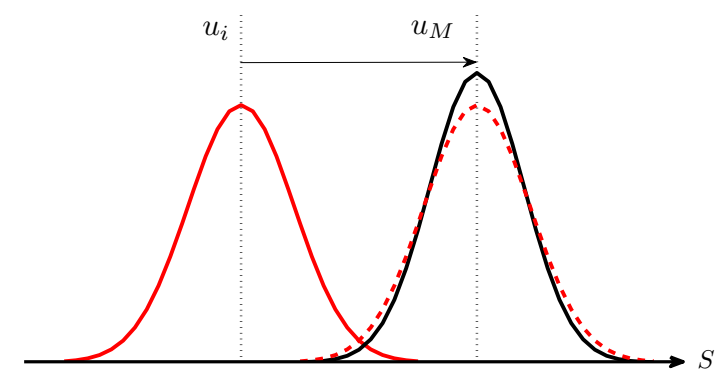

Fig. 4. Naïve Approach

site, denoted as target web site from the pool of web sites to be protected. We manipulate the features of other web sites so that all web sites look like each other in terms of sample mean and sample variance.

This naive countermeasure is discussed in details as follows. First, we choose a web site with the maximum sample mean from the pool of web sites. We presume the sample mean is normally distributed. Denote sample mean of the target web site as $\mu_{M}$. Sample mean of the $\mathrm{i}-t h$ web site is $\mu_{i}$. Then we introduce delay to shift the RTT sample mean of the ith web site close to the RTT sample mean of the target web site so that $\mu_{M}=\mu_{i}$, as illustrated in Figure 4. In this way, we increase the overlapping zone of these two distributions and increase the error rate in Equation (9). According to Theorem 1 , the detection rate will decrease significantly since the two web sites have the same mean now.

However, the analysis of sample mean and sample variance in Section IV-C shows that even if the sample mean of all web sites is the same, sample variance of the RTTs may still disclose the web site. Theorem 2 also demonstrates that when $r$ approaches 1, the web sites can not be identified. Recall from Equation (19), the variance ratio $r=\frac{\sigma_{c}^{2}+\sigma_{p}^{2}+\sigma_{w_{i}}^{2}}{\sigma_{c}^{2}+\sigma_{p}^{2}+\sigma_{w_{j}}^{2}}$. Consequently, we can increase sample variance $\sigma_{p}^{2}$ at the $\mathrm{SSH}$ server to achieve $r \rightarrow 1$. We can carefully select a maximum sample variance $\sigma_{M}^{2}$ which is larger than sample variance of all the web sites and manipulate the delay so that all web sites have the same variance. Therefore, by manipulating web traffic delay, all web sites will have the same distribution, $\mathcal{N}\left(\mu_{M}, \sigma_{M}^{2}\right)$.

We can use the squared Hellinger distance as a metric to estimate the distance between two normal distributions $\mathcal{P} \sim$ $\mathcal{N}\left(\mu_{i}, \sigma_{i}^{2}\right)$ and $\mathcal{Q} \sim \mathcal{N}\left(\mu_{M}, \sigma_{M}^{2}\right)$,

$$
\mathcal{D}_{i}=\mathcal{H}^{2}(\mathcal{P}, \mathcal{Q})=1-\sqrt{\frac{2 \sigma_{M} \sigma_{i}}{\sigma_{M}^{2}+\sigma_{i}^{2}}} e^{-\frac{1}{4} \frac{\left(\mu_{M}-\mu_{i}\right)^{2}}{\sigma_{M}^{2}+\sigma_{i}^{2}}}
$$

The total distance can be calculated as $\mathcal{D}=\sum_{i=1}^{N} \mathcal{D}_{i}$. In this way, the adversary cannot distinguish the web sites visited by users in terms of RTT, and we can ensure the users can achieve the maximum anonymity degree, i.e., $\log N$. However, this naive approach will introduce considerable delay into the web traffic, which significantly affects the web browsing performance.

\section{B. $k$-means Clustering based Approach}

To balance the tradeoff between the performance and anonymity degree, we propose to use the $k$-means algorithm to group web sites into $k$ clusters. Web sites in one cluster have similar means. Denote $t_{1}, t_{2}, \ldots, t_{N}$ as the feature set for $N$ web sites, where $t_{i}$ is a $x$-dimensional feature vector for the $i-t h$ web site. By using the $k$-means clustering, we aim to partition the $N$ web sites into $k$ clusters $\mathcal{C}=\left\{\mathcal{C}_{1}, \mathcal{C}_{2}, \ldots, \mathcal{C}_{k}\right\}$ $(k \leqslant N)$. We choose a feature vector for each cluster and will shape all web sites to have that feature vector. In our case, ¡maximum sample mean, maximum sample variance; of all web sites in a cluster is chosen as the feature vector of that cluster. The corresponding target distributions for these clusters are $\left\{\mathcal{N}\left(\mu_{1}^{\prime}, \sigma_{1}^{\prime}\right), \mathcal{N}\left(\mu_{2}^{\prime}, \sigma_{2}^{\prime}\right), \ldots, \mathcal{N}\left(\mu_{N}^{\prime}, \sigma_{N}^{\prime}\right)\right\}$. Therefore, for the $i-t h$ cluster, we manipulate all web sites to have a distribution of $\left.\mathcal{N}\left(\mu_{N i}^{\prime}, \sigma_{i}^{\prime}\right)\right\}$. Using Equation (25), we can derive the distance $\mathcal{D}_{\rangle}^{\prime}$ between each web site and the target web site for each cluster and obtain the total distance $\mathcal{D}^{\prime}=\sum_{i=1}^{N} \mathcal{D}^{\prime}{ }_{i}$

In the $k$-means based countermeasure, traffic from a web site is shaped to have the distribution of its cluster, instead of a globe distribution as in the naive approach. Consequently, the network delay introduced by the $k$-means clustering based countermeasure can be significantly reduced.

\begin{tabular}{l}
\hline Algorithm $1 \mathbb{K}$-Anonymity Algorithm \\
Require:
\end{tabular}

(a) $\mathcal{C}$, a set of clusters derived by using $k$-means Clustering; (b) $\mathcal{C}_{\mathcal{K}}$, an empty set of clusters to satisfy $k$ anonymity Clustering; (c) $\mathcal{C}_{i}$, certain cluster in $\mathcal{C}$; (c) $\mathcal{C}_{m}$, certain cluster with maximum site number in $\mathcal{C}$; (d) $\mathcal{D}\left(\mathcal{C}_{i}, \mathcal{C}_{j}\right)$, the distance between $\mathcal{C}_{i}$ and $\mathcal{C}_{i}$; (e) $\mathcal{R}$, the remainder when site number is divided by $\mathbb{K}$.

Ensure: $\mathcal{C}$ Satisfies $\mathbb{K}$-Anonymity

1: while Not null in $\mathcal{C}$ do

2: for $\left|\mathcal{C}_{i}\right|<\mathbb{K}$ do

3: $\quad$ Search $\mathcal{C}$ to find $\mathcal{C}_{j}$ such that $\mathcal{D}\left(\mathcal{C}_{i}, \mathcal{C}_{j}\right)$ is minimum.

4: $\quad$ Merger clusters $\mathcal{C}_{i}$ and $\mathcal{C}_{j}$

5: end for

6: $\quad$ for each cluster $\mathcal{C}_{i}$ such that $\left|\mathcal{C}_{i}\right|>2 \mathbb{K}$ do

7: $\quad$ Partition the group into $\left\lceil\frac{\left|\mathcal{C}_{i}\right|}{\mathbb{K}}\right\rceil$ clusters to ensure that $\left\lfloor\frac{\left|\mathcal{C}_{i}\right|}{\mathbb{K}}\right\rfloor$ clusters have at least $\mathbb{K}$ web sites accord to minimum $\mathcal{C}_{i}$ and $\mathcal{C}_{m}$;

8: $\quad$ end for

9: $\quad$ while Exist certain cluster $\left|\mathcal{C}_{i}\right|$ is between $\mathbb{K}$ and $\mathbb{K}+\mathcal{R}$ do

10: $\quad$ Transfer cluster $\left|\mathcal{C}_{i}\right|$ to $\left|\mathcal{C}_{i}\right|<\mathbb{K}$ and adjust $\mathcal{R}$;

11: end while

12: Partition $\left|\mathcal{C}_{i}\right|$ with maximum site number into 2 clusters to ensure that one has at least $\mathbb{K}$ web sites accord to minimum $\mathcal{C}_{i}$ and $\mathcal{C}_{m}$;

13: end while 


\section{C. $\mathbb{K}$-Anonymity based Approach}

The astute reader would surely have noticed that one issue that we have seemingly ignored is the anonymity degree of web sites in each cluster in the introduction of the $k$-means strategy. If the number of web sites in a cluster is very small, the adversary can readily identify the set of web sites visited by a user. For example, if there is only one web site in a cluster, the attacker can definitively identify the web site. Therefore, we shall ensure the anonymity degree for each cluster of web sites.

To address this issue, we introduce $\mathbb{K}$-Anonymity, to guarantee a minimum degree anonymity for all clusters. Particularly, $\mathbb{K}$-Anonymity is defined as if and only if there are at least $\mathbb{K}$ web sites in each cluster. According to the existing partition of the cluster via $k$-means, we use Algorithm 1 to achieve $\mathbb{K}$-Anonymity. We first define the distance between two clusters. Denote the target web site in $\mathcal{C}_{i}$ as $\mathcal{P}^{\prime} \sim \mathcal{N}\left(\mu_{i}^{\prime},\left(\sigma_{i}^{\prime}\right)^{2}\right)$, where $\mu_{i}^{\prime}$ and $\left(\sigma_{i}^{\prime}\right)^{2}$ is the maximum sample mean and sample variance in $\mathcal{C}_{i}$, respectively. As such, let the target web site in $\mathcal{C}_{j}$ be $\mathcal{Q}^{\prime} \sim \mathcal{N}\left(\mu_{j}^{\prime},\left(\sigma_{j}^{\prime}\right)^{2}\right)$. Then we have the distance between $\mathcal{C}_{i}$ and $\mathcal{C}_{j}$ derived by

$$
\mathcal{D}\left(\mathcal{C}_{i}, \mathcal{C}_{j}\right)=\mathcal{H}^{2}\left(\mathcal{P}^{\prime}, \mathcal{Q}^{\prime}\right) .
$$

Then we use an efficient heuristic algorithm Algorithm 1 to achieve $\mathbb{K}$-Anonymity The basic idea is that we choose the cluster $\mathcal{C}_{i}$ and $\left|\mathcal{C}_{i}\right|$ is less than $\mathbb{K}$. Then we search a cluster $\mathcal{C}_{j}$, where $\mathcal{D}\left(\mathcal{C}_{i}, \mathcal{C}_{j}\right)$ is minimum. Therefore, we then merge these two clusters. If the cardinality of the new cluster is larger than $2 \mathbb{K}$, we partition the cluster into $\left\lfloor\frac{\left|\mathcal{C}_{i}\right|}{\mathbb{K}}\right\rfloor$ cluster. Eventually, we can get the final clusters that meet the $\mathbb{K}$-Anonymity requirement.

\section{Vi. Evaluation}

We have implemented the novel RTT based side-channel attack against encrypted web sites. In this section, we use real-world experiments to demonstrate the information leakage caused by the side channel attack and feasibility and effectiveness of countermeasures proposed in Section V.

\section{A. Experiment Setup}

Figure 5 illustrates the experiment setup. We deployed a SSH sever in north America. Two other computers were deployed in Asia. One computer acts as a client and is connected to a wireless access point. The mimic attacking computer sniffs the timing of packets into and out of the client. All computers run Fedora Core 14. The version of OpenSSH [25] at both SSH server and client is $5.8 p 2$. The web browser is Firefox 3.6.17. We configure Firefox not to cache the data. The latest Adobe Flash plugin [26] is installed at the browser.

\section{B. Detection Rate}

To validate the effectiveness of the side channel attack, we target the top 100 web sites in the Alexa rankings [27]. Since the distribution of popularity of the web sites is a Zipf distribution, i.e., $80 \%$ users visit $20 \%$ web sites, this set of web sites are sufficient to demonstrate the potential threat from the network delay based side channel. To access the web sites at the client side, we modify Pagestats [28] and drive Firefox to visit these web sites automatically. The libpcap 1.1.1 library and TCPdump 4.1.1 are used to capture packets. The client automatically accesses each web site one by one and dumps traffic from a web site through TCPdump. The client fetched each web site for 100 times and the experiments lasted for around one month. By using the approach in Section III-A, we can calculate RTTs between each of the top 100 web sites and the client, and establish the profiles for these web sites.

To validate the damage of the side channel attack, we use data collected in odd days as the training set and data collected in even days as the test set. We use the Weka toolkit [29] to implement the classification in Section III-C, with Gaussian kernel density estimation. The result of an experiment is a table of probabilities for each website in the test set. We adopt the $\lambda$-identifiability [4] for an instance. An instance is defined as 1 if the actual class of the instance is in the top $\lambda$ of the predicted class list, as ordered by probability estimate or the instance is defined as $\mathbf{0}$ if the actual class is not in the top $\lambda$.

Figure 6 illustrates the detection rate in terms of different values of $\lambda$. As we can see from this figure, the detection rate increases with $\lambda$. Detection rate achieved by sample mean is better than detection rate sample variance. This shows that the sample mean is a primary factor affecting detection rate. In addition, detection rate archived by the vector of sample mean and sample variance defined in Equation (5) is significantly better than detection rate via a single feature. As demonstrated by our experimental results, the detection rate achieved by the vector of sample mean and sample variance is around $98.7 \%$ when $\lambda=10$. This shows that the information leakage from network delay is a significant threat to user privacy.

\section{Countermeasures}

To validate the effectiveness of our countermeasures, we introduce extra random delay to web traffic at the proxy side. First, we tested the naive approach and manipulated the sample mean of the RTT for each web site so that those web sites have the same (maximum) sample mean. Figure 7 illustrates the detection rate after this manipulation. As we can see from this figure, the detection rate of sample mean is significantly degraded, which is consistent with our analysis in Section V-A. However, the detection rate via sample variance can still leak information to some extent. The vector of sample mean and sample variance produced similar detection rate to sample variance.

Figure 8 gives the relationship between detection rate of sample variance and $\sigma_{p}$ with $\lambda=10$, where $\sigma_{p}$ is defined in Equation (13). By manipulating the standard deviation $\sigma_{p}$ of web traffic at the SSH proxy, the detection rate via sample variance is dramatically degraded. This is consistent with Theorem 2, that is, when $r \rightarrow 1$, the adversary can not differentiate different web sites.

Figure 9 illustrates the relationship between anonymity degree of the smallest cluster and number of the clusters for the k-means countereasure. We used the information leakage 


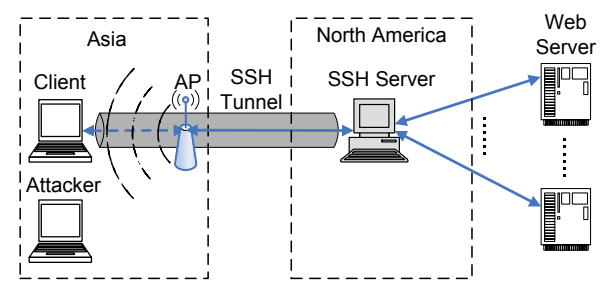

Fig. 5. Experiment Setup

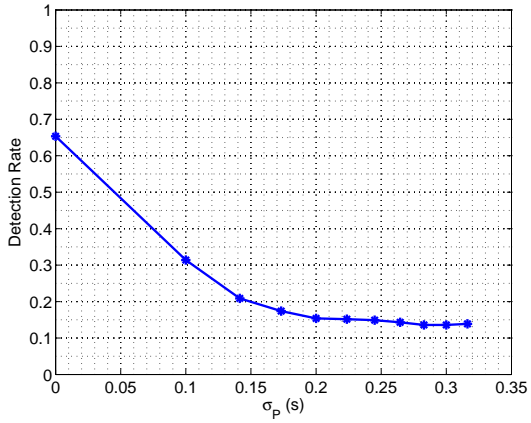

Fig. 8. Effect upon Detection Rate of Sample Variance with $\lambda=10$ by Manipulating $\sigma_{p}$

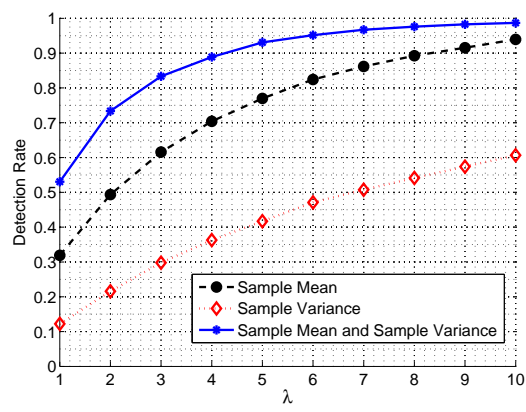

Fig. 6. Detection Rate v.s. Distinct Values of $\lambda$

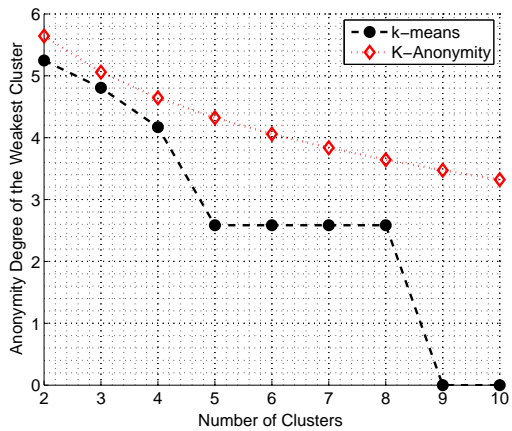

Fig. 9. Anonymity Degree of the Weakest Cluster v.s. Number of Clusters

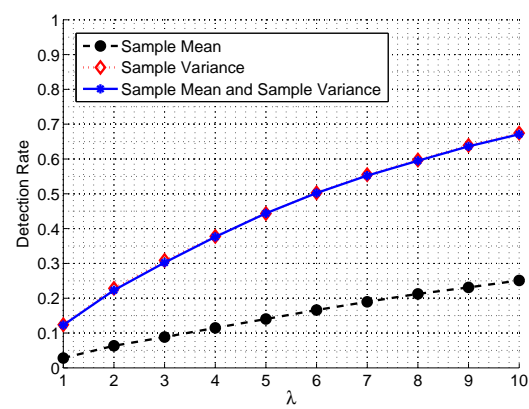

Fig. 7. Detection Rate v.s. Distinct Values of $\lambda$ by Using Statistical Distribution Countermeasure

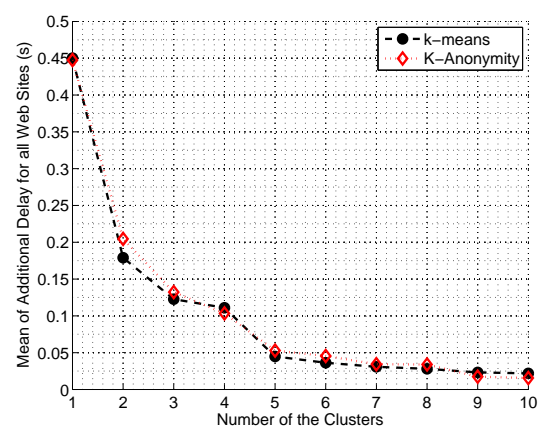

Fig. 10. Mean of Additional Delay for all web sites v.s. Number of Clusters metric defined in Equation (2) to evluate the anonymity degree of the smallest cluster. As we can observe from Figure 9, by using the $k$-means clustering, when $k$ is equal to 9 and 10 , the smallest cluster has only one web site. Therefore, the adversary can readily identify this web site from the rest. the $k$-means clustering fails to preserve user privacy in this case. In comparison, the $\mathbb{K}$-Anonymity based strategy can still prevent the adversary from differentiating web sites from each cluster. When the number of clusters is 10 , the $\mathbb{K}$-Anonymity based strategy achieved an anonymity degree of more than 3 bits (corresponds to 8 websites).

Figure 10 shows the average of extra delay introduced into all web sites. It can be observed when the clustering approach is used, the delay is significantly reduced. Also, we can see that delay added by using the $\mathbb{K}$-Anonymity based approach is similar to the $k$-means based countermeasure while the former achieves a guaranteed anonymity degree. These observations verify that the $\mathbb{K}$-Anonymity based countermeasure can tackle the tradeoff between the performance and anonymity degree for clusters of web sites.

\section{CONCLUSION}

In this paper, we investigated a novel side channel attack via RTTs between a victim user and encrypted web sites. We define detection rate as the probability that an adversary correctly recognizes web sites accessed by a victim user. We conducted careful statistical analysis of different statistics of
RTTs and found that sample mean and sample variance of web traffic RTTs can disclose web sites being accessed. We theoretically analyzed the detection rate and derived closedform formulas. To defend against this attack, we proposed three countermeasures: the naive approach shaping all web traffic to have a similar RTT in terms of mean and variance, the $k$-means strategy grouping web sites with similar RTT mean and variance into the same cluster in order to address the performance issue of the naive approach, and the $k$-anonymity strategy to guarantee a minimum degree of anonymity for web sites in one cluster. We conducted extensive experiments to evaluate the severeness of the side channel attack and feasibility and effectiveness of the countermeasures. Our experiments demonstrate that the $\mathbb{K}$-Anonymity based countermeasure can effectively balance the tradeoff between the performance and information leakage. Our empirical results match our theoretical analysis very well.

\section{REFERENCES}

[1] H. Roberts, E. Zuckerman, J. York, R. Faris, and J. Palfrey, "2010 circumvention tool usage report," http://cyber.law.harvard.edu/sites/cyber. law.harvard.edu/files/2010_Circumvention_Tool_Usage_Report.pdf, 2010.

[2] Q. Sun, D. R. Simon, Y.-M. Wang, W. Russel, V. N. Padmanabhan, and L. Qiu, "Statistical identification of encrypted web browsing traffic," in Proceedings of the IEEE Symposium on Security and Privacy $(S \& P)$, May 2002.

[3] G. D. Bissias, M. Liberatore, D. Jensen, and B. N. Levine, "Privacy vulnerabilities in encrypted http streams," in Proceedings of the 5th Privacy Enhancing Technologies Workshop (PET), May 2005. 
[4] M. Liberatore and B. N. Levine, "Inferring the source of encrypted http connections," in Proceedings of the ACM conference on Computer and Communication Security (CCS), October 2006.

[5] D. Herrmann, R. Wendolsky, and H. Federrath, "Website fingerprinting: attacking popular privacy enhancing technologies with the multinomial naïve-bayes classifier," in Proceedings of the 2009 ACM workshop on Cloud computing security (CCSW), September 2009.

[6] L. Lu, E.-C. Chang, and M. C. Chan, "Website fingerprinting and identification using ordered feature sequences," in Proceedings of the 15th European conference on Research in Computer Security (ESORICS), September 2010.

[7] S. Chen, R. Wang, X. Wang, and K. Zhang, "Side-channel leaks in web applications: a reality today, a challenge tomorrow," in Proceedings of the 31st IEEE Symposium on Security and Privacy $(S \& P)$, May 2010.

[8] C. V. Wright, S. E. Coull, and F. Monrose, "Traffic morphing: An efficient defense against statistical traffic analysis," in Proceedings of the Network and Distributed Security Symposium (NDSS), February 2009.

[9] X. Luo, P. Zhou, E. W. W. Chan, W. Lee, R. K. C. Chang, and R. Perdisci, "Httpos: Sealing information leaks with browser-side obfuscation of encrypted flows," in Proceedings of the 18th Annual Network and Distributed System Security Symposium (NDSS), February 2011.

[10] Y. Zhu, X. Fu, B. Graham, R. Bettati, and W. Zhao, "On flow correlation attacks and countermeasures in mix networks," in Proceedings of Workshop on Privacy Enhancing Technologies (PET), May 2004.

[11] B. N. Levine, M. K. Reiter, C. Wang, and M. Wright, "Timing attacks in low-latency mix-based systems," in Proceedings of Financial Cryptography $(F C)$, February 2004.

[12] D. X. Song, D. Wagner, and X. Tian, "Timing analysis of keystrokes and timing attacks on ssh," in Proceedings of 10th USENIX Security Symposium, August 2001

[13] Q. X. Sun, D. R. Simon, Y. Wang, W. Russell, V. N. Padmanabhan, and L. L. Qiu, "Statistical identification of encrypted web browsing traffic," in Proceedings of IEEE Symposium on Security and Privacy $(S \& P)$, May 2002.

[14] C. V. Wright, L. Ballard, F. Monrose, and G. M. Masson, "Language identification of encrypted voip traffic: Alejandra y roberto or alice and bob?" in Proceedings of the 16th Annual USENIX Security Symposium (Security), August 2007.

[15] C. V. Wright, L. Ballard, S. E. Coull, F. Monrose, and G. M. Masson, "Spot me if you can: Uncovering spoken phrases in encrypted voip conversation." in Proceedings of the 2008 IEEE Symposium on Secrurity and Privacy (S\&P), May 2008.

[16] X. Wang and D. S. Reeves, "Robust correlation of encrypted attack traffic through stepping stones by manipulation of inter-packet delays," in Proceedings of the 2003 ACM Conference on Computer and Communications Security (CCS), November 2003.

[17] X. Wang, S. Chen, and S. Jajodia, "Tracking anonymous peer-to-peer voip calls on the internet," in Proceedings of the 12th ACM Conference on Computer Communications Security (CCS), November 2005.

[18] P. Peng, P. Ning, and D. S. Reeves, "On the secrecy of timing-based active watermarking trace-back techniques," in Proceedings of the IEEE Security and Privacy Symposium (S\&P), May 2006.

[19] W. Yu, X. Fu, S. Graham, D. Xuan, and W. Zhao, "Dsss-based flow marking technique for invisible traceback," in Proceedings of the 2007 IEEE Symposium on Security and Privacy (S\&P), May 2007.

[20] Z. Ling, J. Luo, W. Yu, X. Fu, D. Xuan, and W. Jia, "A new cell counter based attack against tor," in Proceedings of 16th ACM Conference on Computer and Communications Security (CCS), November 2009.

[21] Z. Ling, J. Luo, W. Yu, and X. Fu, "Equal-sized cells mean equal-sized packets in tor?" in Proceedings of Proceedings of ICC Communication and Information System Security Symposium (ICC), June 2011.

[22] Z. Ling, X. Fu, W. Jia, W. Yu, and D. Xuan, "A novel packet size based covert channel attack against anonymizer," in Proceedings of The 30th IEEE International Conference on Computer Communications (INFOCOM), April 2011

[23] A. Pfitzmann and M. Köhntopp, "Anonymity, unobservability and pseudonymity c a proposal for terminology," Designing Privacy Enhancing Technologies, vol. Lecture Notes in Computer Science, LNCS 2009, pp. 1-9, 2001.

[24] Z. Ling, J. Luo, Y. Zhang, X. Fu, and W. Yu, "A novel network delay based side channel attack: Modeling and defense," XXX, Southeast University, Tech. Rep., 2011.

[25] "Openssh," http://www.openssh.com, 2011

[26] "Adobe flash player," http://www.adobe.com/products/flashplayer/, 2011

[27] Alexa Internet, Inc., "Alexa the web information company," http://www. alexa.com/, 2011

[28] S. Dedeo, "Pagestats," http://web.cs.wpi.edu/ cew/pagestats/, 2011.
[29] "Weka 3: Data mining software in java," http://www.cs.waikato.ac.nz/ $\mathrm{ml} /$ weka/, 2011.

\section{Appendix A: Proof of TheOrem 1}

Proof: The distribution of sample mean for a normal distribution $\mathcal{N}\left(\mu, \sigma^{2}\right)$ is still a normal one, that is, $\mathcal{N}\left(\mu, \frac{\sigma^{2}}{n}\right)$. Hence, sample mean $\bar{T}_{i}$ for the web site $w_{i}$ has a normal distribution

$$
f_{i}(t)=\mathcal{N}\left(\mu_{i}, \sigma_{1}^{2}\right)=\mathcal{N}\left(\mu_{i}, \frac{\sigma_{i}^{2}}{n}\right) .
$$

Similarly, sample mean $\bar{X}$ for web site $w_{j}$ has a normal distribution.

$$
f_{j}(t)=\mathcal{N}\left(\mu_{j}, \sigma_{2}^{2}\right)=\mathcal{N}\left(\mu_{j}, \frac{\sigma_{j}^{2}}{n}\right) .
$$

Since $\bar{X}_{i}$ and $\bar{X}_{j}$ are normally distributed, we can use the Bhattacharyya bound to estimate the error as follows:

$$
P_{e}(\bar{x}) \leq \sqrt{P\left(W_{i}\right) P\left(W_{j}\right)} \int \sqrt{f\left(x \mid W_{i}\right) f\left(x \mid W_{j}\right)} d_{x} .
$$

Substituting Equation (27), (28), and $P\left(W_{i}\right)=P\left(W_{j}\right)=0.5$ into Equation (29) and carrying out the integration, we have

$$
P_{e}(\bar{x}) \leq \frac{1}{2} e^{-k}
$$

where

$$
k=1 / 8\left(\mu_{j}-\mu_{i}\right)^{2}\left(\frac{\sigma_{i}^{2}+\sigma_{j}^{2}}{2 n}\right)^{-1}+\frac{1}{2} \ln \frac{\sigma_{i}^{2}+\sigma_{j}^{2}}{2 \sqrt{\sigma_{i}^{2} \sigma_{j}^{2}}} .
$$

After substituting Equation (19) into Equation (31) and some rearranging, we have

$$
k=1 / 4\left(\mu_{j}-\mu_{i}\right)^{2} \frac{n}{\sigma_{i}^{2}+\sigma_{j}^{2}}+\frac{1}{2} \ln \frac{\frac{1}{\sqrt{r}}+\sqrt{r}}{2}
$$

Substituting Equation (32) into Inequality (30), the error rate is given by

$$
P_{e}(\bar{x}) \leq e^{-1 / 4} e^{-\left(\mu_{j}-\mu_{i}\right)^{2}} e^{-\frac{n}{\sigma_{i}^{2}+\sigma_{j}^{2}}} \frac{1}{\sqrt{2\left(\frac{1}{\sqrt{r}}+\sqrt{r}\right)}} .
$$

The detection rate $P_{d}(\bar{X})$ then satisfies

$$
\begin{aligned}
P_{d}(\bar{x}) & =1-P_{e}(\bar{x}) \\
& =1-e^{-\left(\mu_{j}-\mu_{i}\right)^{2} / 4} e^{-\frac{n}{\sigma_{i}^{2}+\sigma_{j}^{2}}} \frac{1}{\sqrt{2\left(\frac{1}{\sqrt{r}}+\sqrt{r}\right)}}
\end{aligned}
$$

Thus, the lower bound of Inequality (34) as the estimation of detection rate by sample mean is derived. The theorem is proven. 